\title{
Inferring periodic orbits from spectra of simply shaped microlasers
}

\author{
M. Lebental, ${ }^{1,2, *}$ N. Djellali, ${ }^{1}$ C. Arnaud, ${ }^{1}$ J.-S. Lauret, ${ }^{1}$ J. Zyss, ${ }^{1}$ R. Dubertrand, ${ }^{2}$ C. Schmit, ${ }^{2, \dagger}$ and E. Bogomolny ${ }^{2}$ \\ ${ }^{1}$ CNRS, Ecole Normale Supérieure de Cachan, UMR 8537, Laboratoire de Photonique Quantique et Moléculaire, \\ 94235 Cachan, France \\ ${ }^{2}$ CNRS, Université Paris Sud, UMR 8626, Laboratoire de Physique Théorique et Modèles Statistiques, 91405 Orsay, France
}

(Received 3 April 2007; published 31 August 2007)

\begin{abstract}
Dielectric microcavities are widely used as laser resonators and characterizations of their spectra are of interest for various applications. We experimentally investigate microlasers of simple shapes (Fabry-Perot, square, pentagon, and disk). Their lasing spectra consist mainly of almost equidistant peaks and the distance between peaks reveals the length of a quantized periodic orbit. To measure this length with a good precision, it is necessary to take into account different sources of refractive index dispersion. Our experimental and numerical results agree with the superscar model describing the formation of long-lived states in polygonal cavities. The limitations of the two-dimensional approximation are briefly discussed in connection with microdisks.
\end{abstract}

DOI: 10.1103/PhysRevA.76.023830

PACS number(s): 42.55.Sa, 05.45.Mt, 03.65.Sq, 42.60.Da

\section{INTRODUCTION}

Two-dimensional microresonators and microlasers are being developed as building blocks for optical telecommunications $[1,2]$. Furthermore, they are of interest as sensors for chemical or biological applications [2-4] as well as billiard toy models for quantum chaos $[5,6]$. Towards fundamental and applied considerations, their spectrum is one of the main features. It was used, for instance, to experimentally recover some information about the refractive index [7] or geometrical parameters [8].

In this paper we focus on cavities much larger than the wavelength and propose to account for spectra in terms of periodic orbit families. Cavities of the simplest and most currently used shapes were investigated: the Fabry-Perot resonator, polygonal cavities such as square and pentagon, and circular cavities.

Our experiments are based on quasi-two-dimensional organic microlasers [9]. The relatively straightforward fabrication process ensures good quality and reproducibility as well as versatility in shapes and sizes (see Fig. 1). The experimental and theoretical approaches developed in this paper can be easily extended to more complicated boundary shapes. Moreover, this method is useful towards other kinds of microresonators, as it depends only on cavity shape and refractive index.

The paper is organized as follows. In Sec. II a description of the two-dimensional model is provided together with its advantages and limitations. In Sec. III microlasers in the form of a long stripe are investigated as Fabry-Perot resonators to test the method and evaluate its experimental precision. This protocol is then further applied to polygonal cavities. In Sec. IV the case of square cavities is discussed whereas in Sec. V dielectric pentagonal cavities are investigated. The theoretical predictions based on a superscar model are compared to experiments as well as numerical

\footnotetext{
*Electronic address: lebental@lpqm.ens-cachan.fr

${ }^{\dagger}$ Deceased.
}

simulations and a good agreement is found. Finally, in Sec. VI the case of several coexisting orbits is briefly dealt with on the example of circular cavities.

\section{PRELIMINARIES}

Dielectric microcavities are quasi-two-dimensional objects whose thickness is of the order of the wavelength but
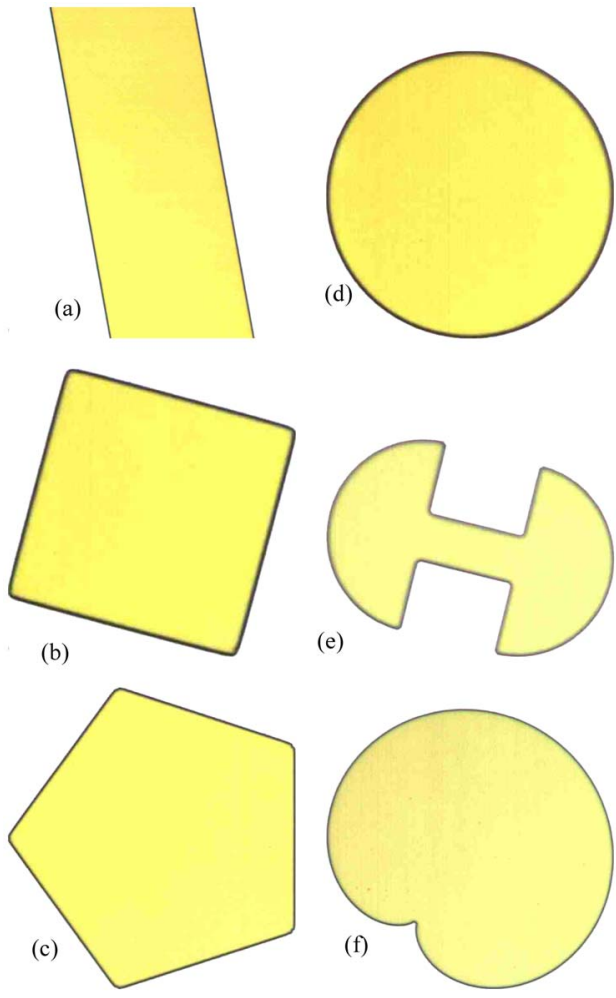

FIG. 1. (Color online) Optical microscope photographs of some organic microlasers: stripe (partial view, used as Fabry-Perot resonator), square, pentagon, disk, quasistadium, and cardioid. Typical dimension: $100 \mu \mathrm{m}$. 


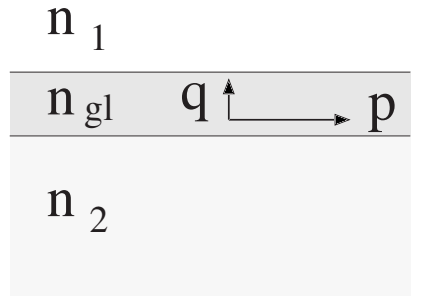

(a)

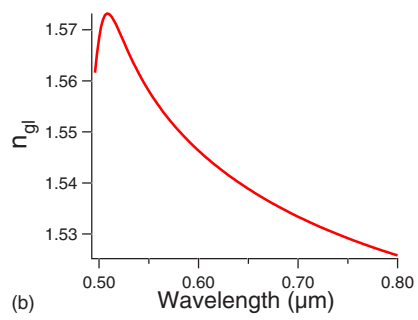

FIG. 2. (Color online) (a) Notations for refractive indexes and propagation wave numbers. From top to bottom, the layers of our samples [9] are the air $\left(n_{1}=1\right)$, a polymer (PMMA) doped with a laser dye (DCM) $\left(n_{g l}=1.54\right)$, and another polymer (SOG) $\left(n_{2}=1.42\right)$ or silica $\left(n_{2}=1.45\right)$. (b) Refractive index of the gain layer vs the wavelength inferred from ellipsometric measurements.

with much bigger plane dimensions (see Fig. 1). Although such cavities have been investigated for a long time both with and without lasing, their theoretical description is not quite satisfactory. In particular, the authors are not aware of true three-dimensional studies of high-excited electromagnetic fields even for passive cavities. Usually one uses a two-dimensional approximation but its validity is not under control.

Within such approximation fields inside the cavity and close to its two-dimensional boundary are treated differently. In the bulk, one considers electromagnetic fields as propagating inside an infinite dielectric slab (gain layer) with refractive index $n_{g l}$ surrounded by medias with refractive indices $n_{1}$ and $n_{2}$ smaller than $n_{g l}$. In our experiments, the gain layer is made of a polymer (PMMA) doped with a laser dye (DCM) and sandwiched between the air and a polymer (SOG) layer [see Fig. 2(a) and [9]]. It is well known (see, e.g., [10] or [11]) that in such geometry there exist a finite number of propagating modes confined inside the slab by total internal reflection. The allowed values of transverse momentum inside the slab, $q$, are determined from the standard relation

$$
e^{2 i h q} r_{1} r_{2}=1
$$

where $h$ is the slab thickness and $r_{1,2}$ are the Fresnel reflection coefficients on the two horizontal interfaces. For total internal reflection

$$
r_{i}=\exp \left(-2 i \delta_{i}\right)
$$

where

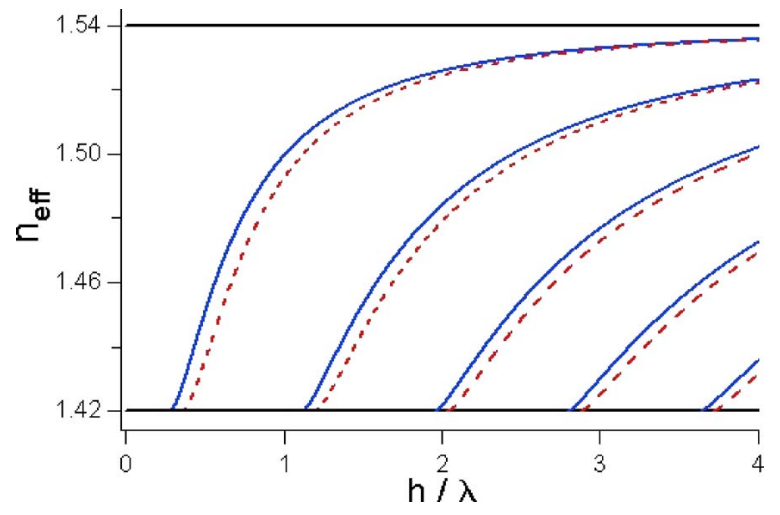

FIG. 3. (Color online) Effective refractive index vs the thickness over wavelength variable calculated from Eq. (4). The refractive indices are assumed to be constant: 1 for air, 1.42 for SOG, and 1.54 for the gain layer (horizontal black lines). The TE polarization is plotted with solid blue lines and TM polarization with dotted red lines. Integer $l$ [see Eq. (4)] increases from left to right starting from $l=0$.

$$
\delta_{i}=\arctan \left(\nu_{i} \frac{\sqrt{n_{g l}^{2} \sin ^{2} \theta-n_{i}^{2}}}{n_{g l} \cos \theta}\right) .
$$

Here $\theta$ is the angle between the direction of wave propagation inside the slab and the normal to the interface. The $\nu_{i}$ parameter is 1 [respectively $\left.\left(n_{g l} / n_{i}\right)^{2}\right]$ when the magnetic field (respectively the electric field) is perpendicular to the slab plane. The first and second cases correspond respectively to TE and TM polarizations.

Denoting the longitudinal momentum, $p=n_{g l} k \sin \theta$, as $p=n_{\text {eff }} k$, the effective refractive index $n_{\text {eff }}$ is determined from the following dispersion relation:

$$
\begin{aligned}
2 \pi \frac{h}{\lambda} \sqrt{n_{g l}^{2}-n_{e f f}^{2}}= & \arctan \left(\nu_{1} \frac{\sqrt{n_{e f f}^{2}-n_{1}^{2}}}{\sqrt{n_{g l}^{2}-n_{e f f}^{2}}}\right) \\
& +\arctan \left(\nu_{2} \frac{\sqrt{n_{e f f}^{2}-n_{2}^{2}}}{\sqrt{n_{g l}^{2}-n_{e f f}^{2}}}\right)+l \pi, \quad l \in \mathbb{N} .
\end{aligned}
$$

This equation has only a finite number of propagating solutions which can easily be obtained numerically. Figure 3 presents possible propagating modes for our experimental setting $n_{1}=1$ (air), $n_{2}=1.42$ (SOG) [12], and $n_{g l}=1.54$ deduced from ellipsometric measurements [see Fig. 2(b)] in the observation range.

The Maxwell equations for waves propagating inside the slab are thus reduced to the two-dimensional scalar Helmholtz equation:

$$
\left(\Delta+n_{e f f}^{2} k^{2}\right) \Psi_{i n}(x, y)=0 .
$$

$\Psi$ represents the field perpendicular to the slab, i.e., the electric field for TM and the magnetic field for TE polarization [13].

This equation adequately describes the wave propagation inside the cavity. But when one of these propagating modes hits the cavity boundary, it can partially escape from the 
cavity and partially be reflected inside it. To describe correctly different components of electromagnetic fields near the boundary, the full solution of the three-dimensional vectorial Maxwell equations is required, which to the authors knowledge has not yet been addressed in this context. Even the much simpler case of scalar scattering by a half plane plate with a small but finite thickness is reduced only to numerical solution of the Wiener-Hopf-type equation [14].

To avoid these complications, one usually considers that the fields can be separated into TE and TM polarization and obey the scalar Helmholtz equations (5),

$$
\left(\Delta+n_{\text {in,out }}^{2} k^{2}\right) \Psi_{\text {in,out }}(x, y)=0,
$$

where $n_{i n}$ is the $n_{\text {eff }}$ effective index inferred from Eq. (4) and $n_{\text {out }}$ is the refractive index of the surrounding media, usually air so $n_{\text {out }}=1$. This system of two-dimensional equations is closed by imposing the following boundary conditions:

$$
\left.\Psi_{\text {in }}\right|_{B}=\left.\Psi_{\text {out }}\right|_{B},\left.\quad \nu_{\text {in }} \frac{\partial \Psi_{\text {in }}}{\partial \vec{\tau}}\right|_{B}=\left.\nu_{\text {out }} \frac{\partial \Psi_{\text {out }}}{\partial \vec{\tau}}\right|_{B} .
$$

Here $\vec{\tau}$ indicates the direction normal to the boundary and $\nu$ depends on the polarization. When the electric (respectively magnetic) field is perpendicular to the cavity plane, called TM polarization (respectively TE polarization), $\nu_{\text {in,out }}=1$ (respectively $\left.\nu_{\text {in,out }}=1 / n_{\text {in,out }}^{2}\right)$. Notice that these definitions of $\nu$ are not the same for horizontal and vertical interfaces.

We consider this standard two-dimensional approach keeping in mind that waves propagating close to the boundary (whispering gallery modes) may deviate significantly from two-dimensional predictions. In particular leakage through the third dimension could modify the lifetime estimation of quasistationary states.

Our polymer cavities are doped with a laser dye and uniformly pumped one by one from above [9], so that the pumping process induces no mode selection. The complete description of such lasing cavities requires the solution of the nonlinear Maxwell-Bloch equations (see, e.g., [15-17] and references therein). For clarity, we accept here a simplified point of view (see, e.g., [1], Sec. 24) according to which true lasing modes can be represented as a linear combination of the passive modes which may lase (i.e., for which gain exceeds losses),

$$
\Psi_{\text {lasing }}=\sum_{m} C_{m} \Psi_{m}
$$

From physical considerations, it is natural to consider the $\Psi_{m}$ modes as the quasistationary states of the passive cavity. Though this choice leads to well-known difficulties (see, e.g., [1]) it is widely noticed and accepted at least for modes with small losses (cf. [16-18]).

For each individual lasing mode, the $C_{m}$ coefficients could be determined only after the solution of the full MaxwellBloch equations. But due to the statistical nature of fluorescence the lasing effect starts randomly and independently during each pump pulse. So it is quite natural to average over many pump pulses. Then the mean spectrum exhibits peaks at frequencies of all possible lasing modes. The experimental
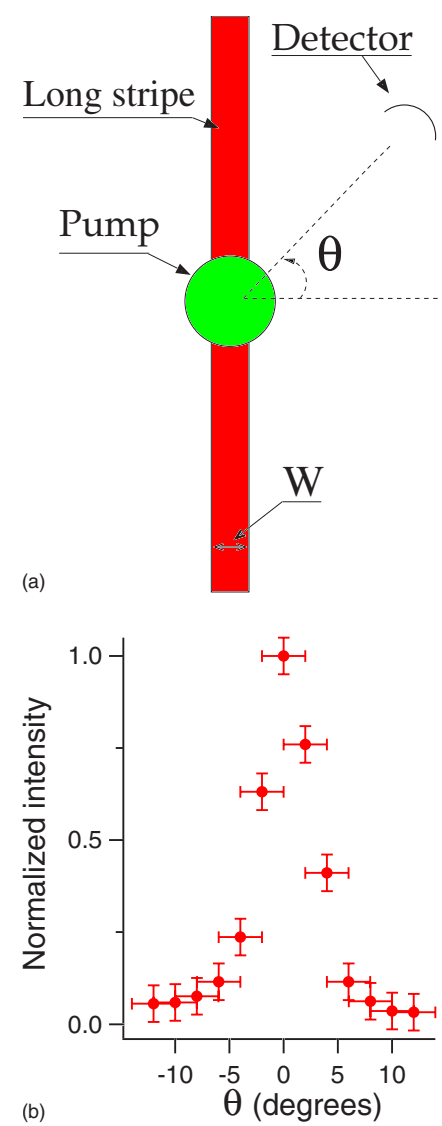

FIG. 4. (Color online) (a) Diagram summarizing the main features of the Fabry-Perot experiment. (b) Detected intensity vs $\theta$ angle for a Fabry-Perot experiment.

data studied in this paper are recorded after integration over 30 pump pulses and agree with this simple statistical model. More refined verifications are in progress.

\section{FABRY-PEROT RESONATOR}

The Fabry-Perot configuration is useful for the calibration control of further spectral experiments due to the nonambiguous single periodic orbit family which sustains the laser effect.

A long stripe can be considered to a good approximation as a Fabry-Perot resonator. In fact, the pumping area is very small compared to the length [see Fig. 4(a)] and the material is slightly absorbing, so that reflections at far extremities can be neglected. Moreover, the pumping area is larger than the width of the stripe, thus the gain is uniformly distributed over the section. For a Fabry-Perot cavity, the emission is expected along both $\theta=0$ and $\theta=\pi$ directions [see Fig. 4(a) for notations]. Figure 4(b) shows that this directional emission is observed experimentally which confirms the validity of our setup.

The experimental spectrum averaged over 30 pump pulses is made up of almost regularly spaced peaks [see Fig. 5(a)] which is typically expected for a Fabry-Perot resonator. In fact, due to coherent effects, the $k$ wave numbers of quasibound states of a passive Fabry-Perot cavity are determined 

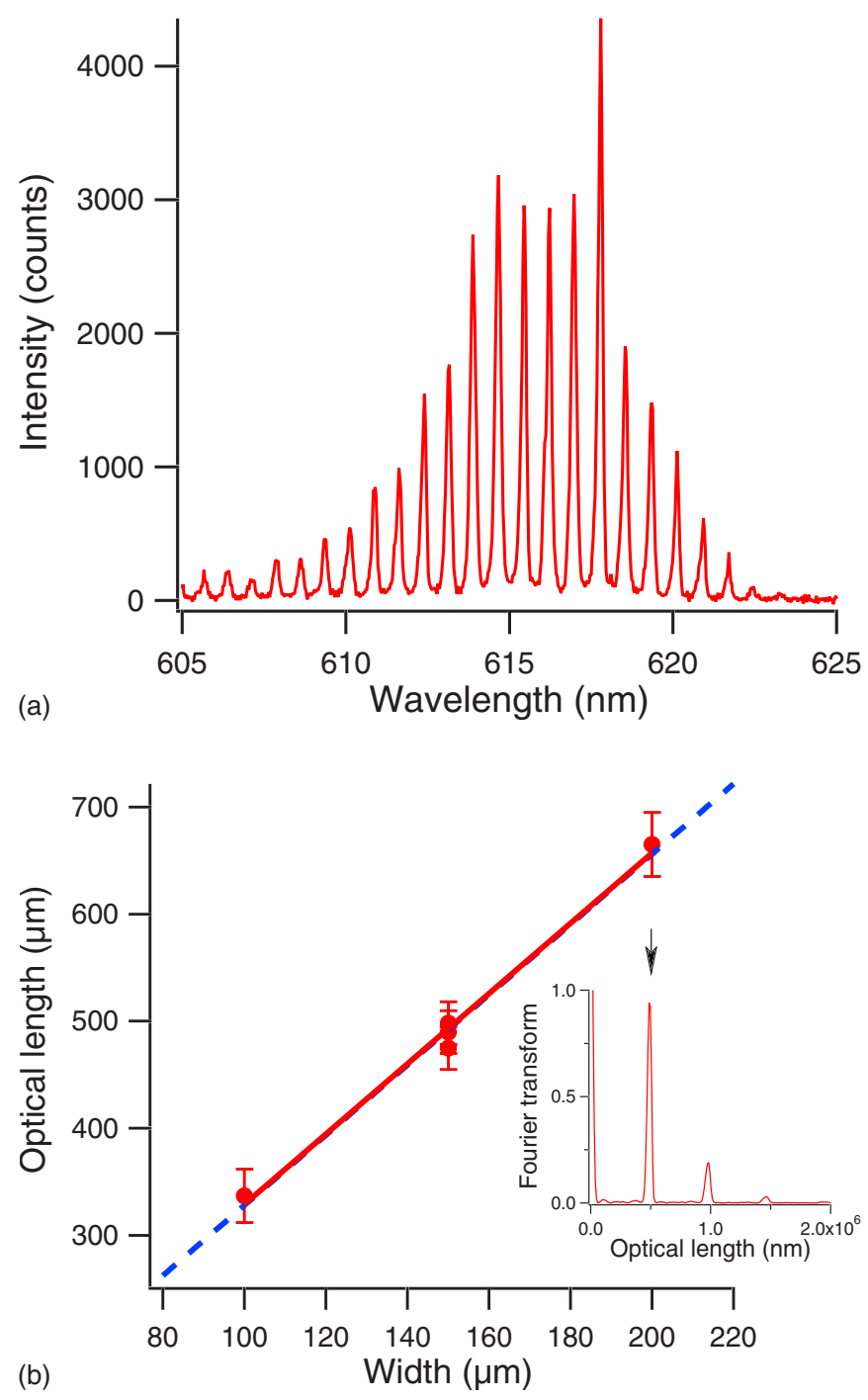

FIG. 5. (Color online) (a) Experimental spectrum of a FabryPerot resonator with $W=150 \mu \mathrm{m}$. (b) Optical length vs Fabry-Perot width $W$. The experiments (red points) are linearly fitted by the solid red line. The dashed blue line corresponds to the theoretical prediction without any adjusted parameter. Inset: Normalized Fourier transform of the spectrum in (a) expressed as intensity vs wave number.

from the quantization condition along the only periodic orbit of $L=2 W$ length as for Eq. (1):

$$
r^{2} e^{i L k n_{e f f}(k)}=1,
$$

where $r$ is the Fresnel reflection coefficient and $n_{\text {eff }}$ is the effective refractive index (4). The solutions of this equation are complex numbers: the imaginary part corresponds to the width of the resonance and the real part (called $k_{m}$ afterwards) gives the position of a peak in the spectrum and verifies

$$
L k_{m} n_{e f f}\left(k_{m}\right)=2 \pi m, \quad m \in \mathbb{N} .
$$

With $\delta k_{m}=k_{m+1}-k_{m}$ assumed to be small, the distance between adjacent peaks is constrained by

$$
\delta k_{m}\left(n_{e f f}\left(k_{m}\right)+k_{m} \frac{\partial n_{e f f}}{\partial k}\left(k_{m}\right)\right) L=2 \pi
$$

We call

$$
n_{\text {full }}=n_{\text {eff }}\left(k_{m}\right)+k_{m} \frac{\partial n_{\text {eff }}}{\partial k}\left(k_{m}\right)
$$

the full effective refractive index. It is a sum over two terms: one corresponding to the phase velocity, $n_{\text {eff }}\left(k_{m}\right)$, and the other one to the group velocity, $k_{m} \frac{\partial n_{\text {eff }}}{\partial k}\left(k_{m}\right)$. If $n_{f u l l}$ is considered as a constant over the observation range, which is true with a good accuracy, $\delta k$ can be retrieved from the experimental spectrum. For instance, the Fourier transform of the spectrum (intensity vs $k$ ) is made up of regularly spaced peaks [Fig. 5(b) inset], with the first one (indicated with an arrow) centered at the optical length $\left(L n_{\text {full }}\right)$ and the others at its harmonics. So the geometrical length of the periodic orbit can be experimentally inferred from the knowledge of $n_{\text {full }}$ which is independently determined as described below. For the Fabry-Perot resonator, the geometrical length is known to be $2 W$, thus allowing us to check the experimental precision. The relative statistical errors on the $W$ width is estimated to be less than 3\%. The error bars in Fig. 5(b) are related to the first peak width of the Fourier transform and are less than 5\% of the optical length.

The full effective refractive index $n_{\text {full }}$ is independently inferred from ellipsometric measurement [Fig. 2(b)] and standard effective index derivation described in the previous section. Depending on the parameter $h / \lambda$ (thickness over wavelength), one or several modes are allowed to propagate. Our samples are designed such as only one TE and TM modes exist with $n_{\text {eff }}$ effective refractive index according to Eq. (4).

In Fig. 3 the refractive index of the gain layer, $n_{g l}$, is assumed to be constant: $n_{g l}=1.54$ in the middle of the experimental window, $\lambda$ varying from 0.58 to $0.65 \mu \mathrm{m}$. From Eq. (4) a $n_{\text {eff }}=1.50$ is obtained in the observation range with a $h=0.6-\mu \mathrm{m}$ thickness, and corresponds to the phase velocity term. The group velocity term $k_{m} \frac{\partial n_{\text {eff }}}{\partial k}\left(k_{m}\right)$ is made up of two dispersion contributions: one from the effective index (about $4 \%$ ) and the other from the gain medium (about 7\%). The dependance of $n_{g l}$ with the wavelength is determined with the GES 5 SOPRA ellipsometer from a regression with the WINELLI II software (correlation coefficient: 0.9988) and plotted in Fig. 2(b). Taking into account all contributions (that means calculating the effective refractive index with a dispersed $\left.n_{g l}\right)$, the $n_{\text {full }}$ full effective refractive index is evaluated to be $1.645 \pm 0.008$ in the observation range. So the group velocity term made up of the two types of refractive index dispersion contribute for $10 \%$ to the full effective index, which is significant compared to our experimental precision. The $n_{\text {full }}$ index depends only smoothly on polarization (TE or TM), and on the $h$ thickness, which is measured with a surface profilometer Veeco (Dektak $\left.{ }^{3} \mathrm{ST}\right)$. Thus the samples are designed with thickness $0.6 \mu \mathrm{m}$ and the precision is reported on the full effective index which is assumed to be 1.64 with a relative precision of about $1 \%$ throughout this work. 
Considering all of these parameters, we obtain a satisfactory agreement between measured and calculated optical lengths, which further improves when taking into account several Fabry-Perot cavities with different widths as shown on Fig. 5(b). The excellent reproducibility (time to time and sample to sample) is an additional confirmation of accuracy and validity. With these Fabry-Perot resonators, we have demonstrated a spectral method to recover the geometrical length of a periodic orbit which can now be confidently applied to different shapes of microcavities.

\section{SQUARE MICROCAVITY}

In the context of this paper square-shaped microcavities present a double advantage. First, they are increasingly used in optical telecommunications $[2,19]$. Second, the precision and validity of the parameters used above can be tested independently since there is only one totally confined periodic orbit family. In fact the refractive index is quite low (about 1.5), so the diamond [see Fig. 6(b), top inset] is the only short-period orbit without refraction loss [i.e., all reflection angles at the boundary are larger than the critical angle $\left.\chi_{c}=\arcsin (1 / n) \approx 42^{\circ}\right]$.

In a square-shaped cavity light escapes mainly at the corners due to diffraction. Thus the quality design of corners is critical for the directionality of emission but not for the spectrum. Indeed for a reasonably well designed squared microcavity (see Fig. 1), no displacement of the spectrum peaks is detectable by changing the $\theta$ observation angle. The spectra used in this paper are thus recorded in the direction of maximal intensity.

Figure 6(a) presents a typical spectrum of a square-shaped microcavity. The peaks are narrower than in the Fabry-Perot resonator spectrum, indicating a better confinement, as well as regularly spaced, revealing a single periodic orbit. Data processing is performed exactly as presented in the previous section: for each cavity the Fourier transform of the spectrum is calculated [Fig. 6(b), bottom inset] and the position of its first peak is located at the optical length. Figure 6(b) summarizes the results for about 20 different microsquares, namely the optical length inferred from the Fourier transform vs the $a$ square side width. These experimental results are fitted by the solid red line. The dotted blue line corresponds to an a priori slope given by $n_{\text {full }}(1.64)$ times the geometrical length of the diamond periodic orbit $(L=2 \sqrt{2} a)$. The excellent agreement confirms that the diamond periodic orbit family provides a dominant contribution to the quantization of dielectric square resonator.

This result is far from obvious as square dielectric cavities are not integrable. At first glance the observed dominance of one short-period orbit can be understood from general considerations based on trace formulas which are a standard tool in semiclassical quantization of closed multidimensional systems (see, e.g., $[20,21]$ and references therein). In general, trace formulas express the density of states (and other quantities as well) as a sum over classical periodic orbits. For two-dimensional closed cavities

$$
d(k) \equiv \sum_{n} \delta\left(k-k_{n}\right) \approx \sum_{p} c_{p} e^{i k L_{p}-i \mu_{p}}+\text { c.c. },
$$

where $k$ is the wave number and $k_{n}$ are the eigenvalues of a closed cavity. The summation on the right part is performed
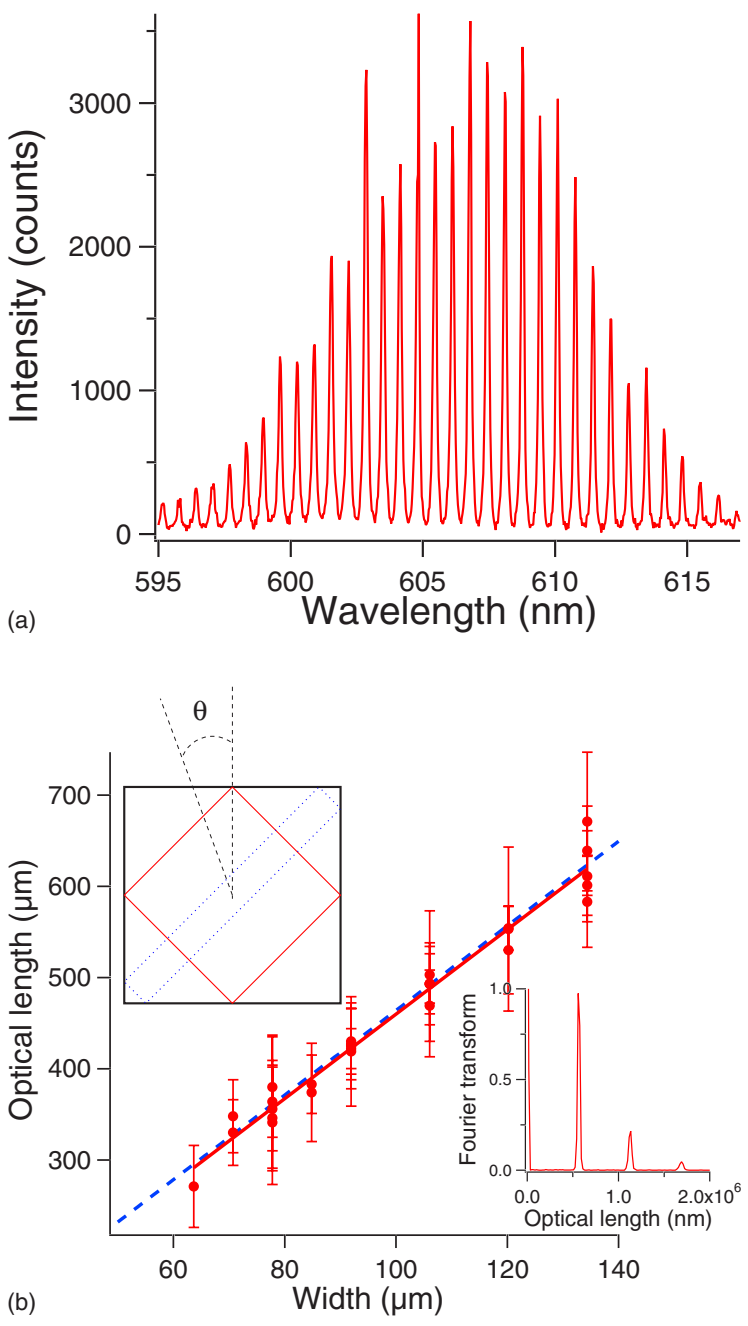

FIG. 6. (Color online) (a) Experimental spectrum of a squareshaped microlaser of $135-\mu \mathrm{m}$ side width. (b) Optical length vs $a$ square side width. The experiments (red points) are linearly fitted by the solid red line. The dashed blue line corresponds to the theoretical prediction (diamond periodic orbit) without any adjusted parameter. Top inset: Two representations of the diamond periodic orbit. Bottom inset: Normalized Fourier transform of the spectrum in (a) expressed as intensity vs wave number.

over all periodic orbits labeled by $p . L_{p}$ is the length of the $p$ periodic orbit, $\mu_{p}$ is a certain phase accumulated from reflection on boundaries and caustics, and amplitude $c_{p}$ can be computed from classical mechanics. In general, for integrable and pseudointegrable systems (e.g., polygonal billiards) classical periodic orbits form continuous periodic orbit families and in two dimensions

$$
c_{p} \sim \frac{A_{p}}{\sqrt{L_{p}}},
$$

where $A_{p}$ is the geometrical area covered by a periodic orbit family (see the example of circular cavities in Sec. VI).

Nonclassical contributions from diffractive orbits and different types of creeping waves (in particular, lateral waves [21]) are individually smaller by a certain power of $1 / k$ and 
are negligible in the semiclassical limit $k \rightarrow \infty$ compared to periodic orbits.

There exist no true bound states for open systems. One can only compute the spectrum of complex eigenfrequencies of quasistationary states. The real parts of such eigenvalues give the positions of resonances and their imaginary parts measure the losses due to the leakage from the cavity.

For such systems it is quite natural to assume that the density of quasistationary states

$$
d(k) \equiv \frac{1}{\pi} \sum_{n} \frac{\operatorname{Im}\left(k_{n}\right)}{\left[k-\operatorname{Re}\left(k_{n}\right)\right]^{2}+\operatorname{Im}\left(k_{n}\right)^{2}}
$$

can be written in a form similar to Eq. (13) but the contribution of each periodic orbit has to be multiplied by the product of all reflection coefficients along this orbit (as it was done in a slightly different problem in [21]),

$$
d(k) \approx \sum_{p} c_{p}\left[\prod_{j=1}^{N_{p}} r_{p}^{(j)}\right] e^{i k L_{p}-i \mu_{p}}+\text { c.c. }
$$

Here $N_{p}$ is the number of reflections at the boundary and $r_{p}^{(j)}$ is the value of reflection coefficient corresponding to the $j$ th reflection for the $p$ periodic orbit.

When the incident angle is larger than the critical angle the modulus of the reflection coefficient equals 1 [see Eq. (2)], but if a periodic orbit hits a piece of boundary with an angle smaller than the critical angle, then $\left|r_{p}\right|<1$ thus reducing the contribution of this orbit. Therefore the dominant contribution to the trace formula for open dielectric cavities is given by short-period orbits $\left(c_{p} \propto 1 / \sqrt{L_{p}}\right)$ which are confined by total internal reflection. For a square cavity with $n=1.5$ the diamond orbit is the only confined short-period orbit which explains our experimental observation of its dominance.

Nevertheless, this reasoning is incomplete because the summation of contributions of one periodic orbit and its repetitions in polygonal cavities does not produce a complex pole which is the characteristic of quasistationary states.

In order to better understand the situation, we have performed numerical simulations for passive square cavities in a two-dimensional approximation with TM polarization (see Sec. II and [22]). Due to symmetries, the quasistationary eigenstates can be classified according to different parities with respect to the square diagonals. In Fig. 7(a), the imaginary parts of wave numbers are plotted vs their real part for states antisymmetric according to the diagonals (that means obeying the Dirichlet boundary conditions along the diagonals) and called here (- -) states.

These quasistationary states are clearly organized in families. This effect is more pronounced when wave functions corresponding to each family are calculated. For instance, wave functions for the three lowest families with (- -) symmetry are presented in the top of Fig. 8. The other members of these families have similar patterns. The existence of such families was first noted in [23] for hexagonal dielectric cavities, then further detailed in [22].
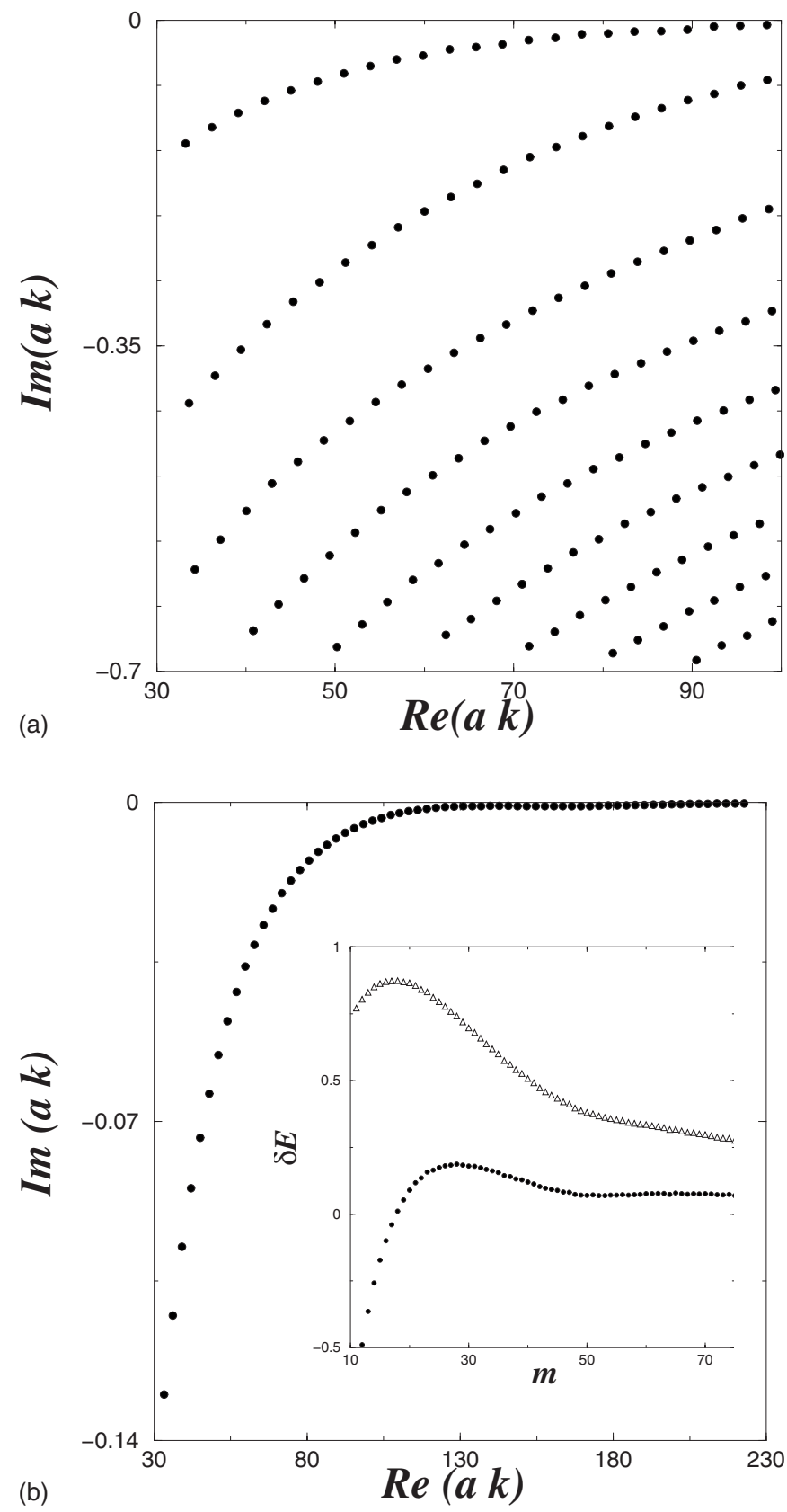

FIG. 7. (a) Imaginary parts vs real parts of the wave numbers of quasistationary states with (- -) symmetry for a dielectric square resonator with $n_{\text {eff }}=1.5$ surrounded by air with $n=1$. (b) The same as in (a) but for the states with the smallest modulus of the imaginary part (the most confined states). Inset. Empty triangles: the difference (20) between the real part of these wave numbers and the asymptotic expression. Filled circles: the same but when the correction term (21) is taken into account.

One can argue that the origin of such families is analogous to the formation of superscar states in pseudointegrable billiards discussed in [24] and observed experimentally in microwave experiments in [25]. In general, periodic orbits of polygonal cavities form continuous families which can be considered as propagating inside straight channels obtained by unfolding classical motion. These channels (hatched area Fig. 9) are restricted by straight lines passing through cavity 


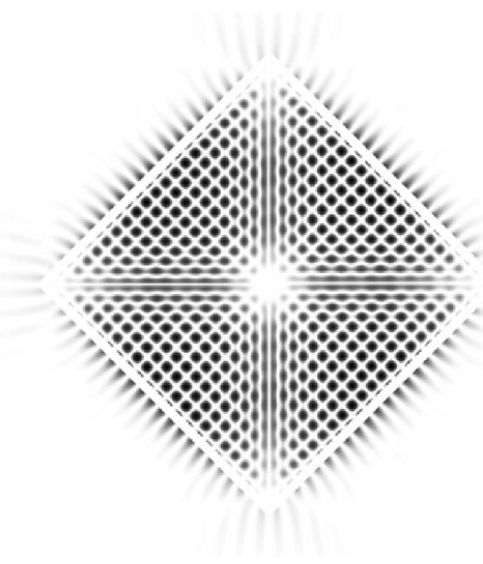

(a)

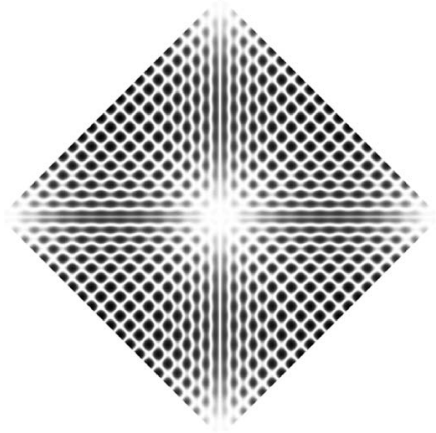

(d)

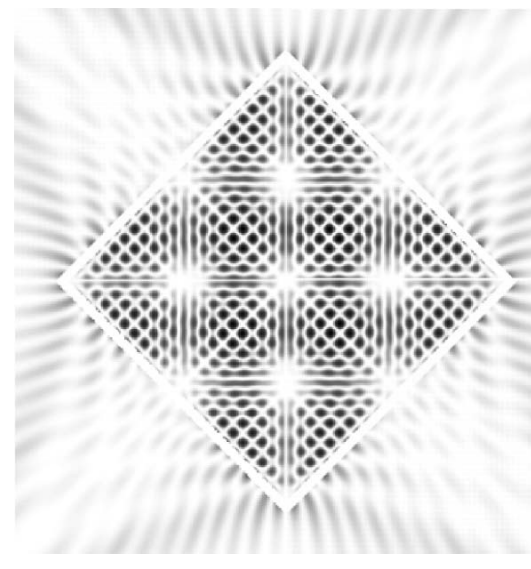

(b)

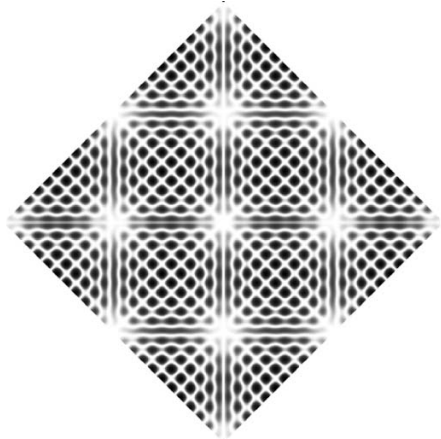

(e)

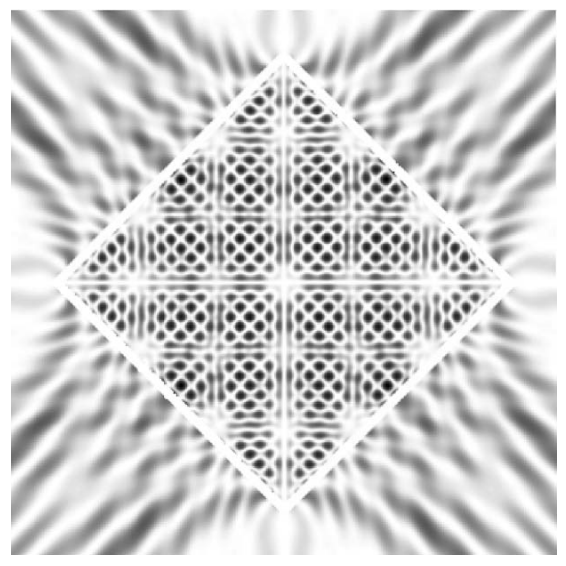

(c)

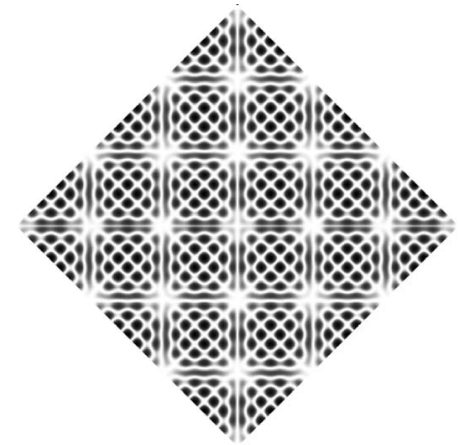

(f)

FIG. 8. (Top) Squared modulus of wave functions with - - symmetry calculated with numerical simulations. (a) $a k=68.74-0.026 \mathrm{i},(\mathrm{b})$ $a k=68.84-0.16 \mathrm{i}$, (c) $a k=69.18-0.33 \mathrm{i}$. (Bottom) Squared modulus of wave functions calculated withing the superscar model (- -) and corresponding to the parameters of the top pictures.

corners. In [24] it was demonstrated that strong quantummechanical diffraction on these singular corners forces wave functions in the semiclassical limit to obey simple boundary conditions on these (fictitious) channel boundaries. More precisely it was shown that for billiard problems $\Psi$ on these boundaries take values of the order of $\mathcal{O}(1 / \sqrt{k}) \rightarrow 0$ when

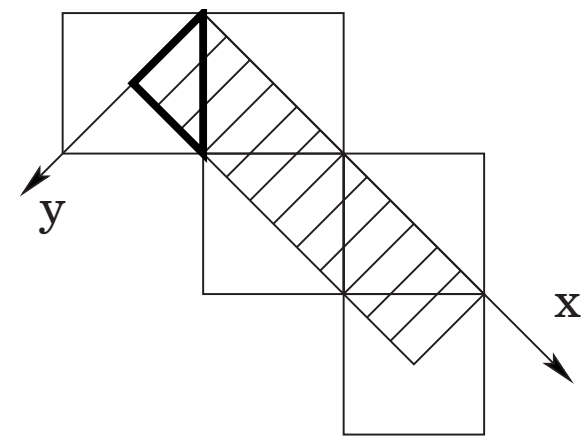

FIG. 9. Unfolding of the diamond periodic orbit. Thick lines indicate the initial triangle. $k \rightarrow \infty$. This result was obtained by using the exact solution for the scattering on periodic array of half planes. No such results are known for dielectric problems. Nevertheless, it seems natural from semiclassical considerations that a similar phenomenon should appear for dielectric polygonal cavities as well.

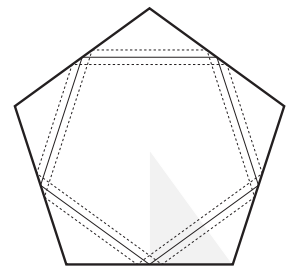

(a)

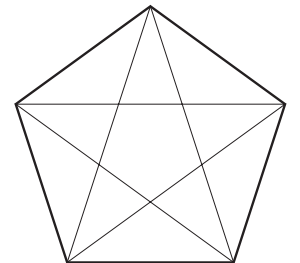

(b)
FIG. 10. (a) Wave numbers for a pentagonal cavity. $a$ is the side length of the cavity. The three most confined families are indicated by solid, dashed, and long-dashed lines. (b) The difference (28) between the real part of quasienergies and superscar expression (25) for the three indicated families in (a). 

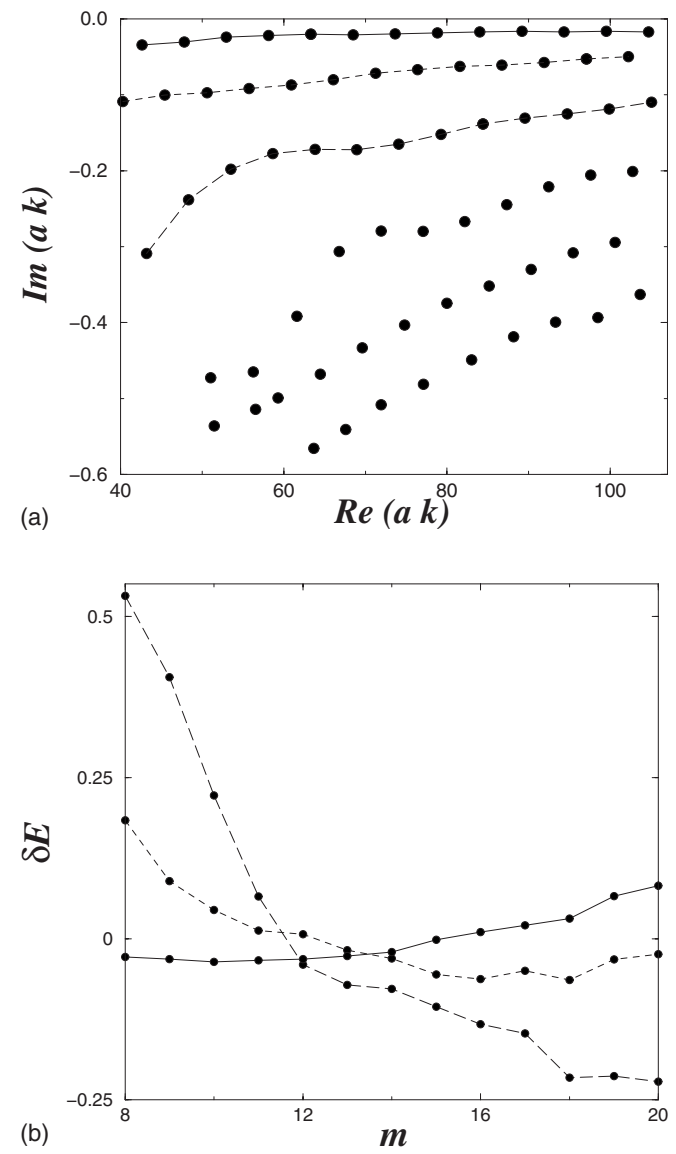

FIG. 11. (a) Wave numbers for a pentagonal cavity. $a$ is the side length of the cavity. The three most confined families are indicated by solid, dashed, and long-dashed lines. (b) The difference between the real part of quasi-energies and superscar expression for the three indicated families in (a).

Within such framework, a superscar state can be constructed explicitly as follows. After unfolding (see Fig. 9), a periodic orbit channel has the form of a rectangle. Its length equals the periodic orbit length and its width is determined by the positions of the closest singular corners. The unfolded superscar state corresponds to a simple plane wave propagating inside the rectangle taking into account all phase changes. It cancels at the fictitious boundaries parallel to the $x$ direction and is periodic along this direction with a periodicity imposed by the chosen symmetry class. This procedure sets the wave number of the state and the true wave function is obtained by folding back this superscar state.

Superscar wave functions with (- -) symmetry associated with the diamond orbit (see Fig. 9) are expressed as follows:

$$
\begin{aligned}
\Psi_{m, p}^{(--)}(x, y)= & \sin \left(\kappa_{m}^{(-)} x\right) \sin \left(\frac{2 \pi}{l} p y\right) \\
& +\sin \left(\kappa_{m}^{(-)} x^{\prime}-2 \delta\right) \sin \left(\frac{2 \pi}{l} p y^{\prime}\right),
\end{aligned}
$$

where $x^{\prime}$ and $y^{\prime}$ are coordinates symmetric with respect to the square side. In coordinates as in Fig. 9,

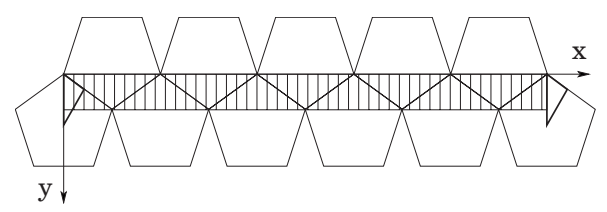

FIG. 12. Unfolding of the five-star periodic orbit for a pentagonal cavity. Thick lines indicate the initial triangle.

$$
x^{\prime}=y, \quad y^{\prime}=x .
$$

In Eq. (17) $m$ and $p$ are integers with $p=1,2, \ldots$, and $m \gg 1 . l=\sqrt{2} a$ is half of the diamond periodic orbit length [26], $\delta$ is the phase of the reflection coefficient defined by $r=\exp (-2 i \delta)$. For simplicity, we ignore slight changes of the reflection coefficient for different plane waves in the functions above. So $\delta$ is given by Eq. (3) with $\nu=1$ for TM polarization and $\theta=\pi / 4$, and $\kappa_{m}^{(-)}$is the momentum defined by

$$
\kappa_{m}^{(-)} l-4 \delta=2 \pi m
$$

This construction conducts to the following expression for the real part of the wave numbers [27]:

$$
\begin{aligned}
n_{e f f} l \operatorname{Re}\left(k_{m, p}\right) & =2 \pi \sqrt{\left(m+\frac{2}{\pi} \delta\right)^{2}+p^{2}} \\
& =2 \pi\left(m+\frac{2}{\pi} \delta\right)+\mathcal{O}\left(\frac{1}{m}\right) .
\end{aligned}
$$

To check the accuracy of the above formulas we plot in the bottom of Fig. 8 scar wave functions (17) with the same parameters as those in the top of Fig. 8. The latter were computed numerically by direct solving the Helmholtz equations (6) but the former looks very similar which supports the validity of the superscar model.

The real part of the wave numbers is tested too. In Fig. 7(b) the lowest loss states (with the smallest modulus of the imaginary part) with (- -) symmetry are presented over a larger interval than in Fig. 7(a). The real parts of these states are compared to superscar predictions (19) with $p=1$, leading to a good agreement. To detect small deviations from the theoretical formula, we plot in the inset of Fig. 7(b) the difference between a quantity inferred from numerical simulations and its superscar prediction from Eq. (19),

$$
\delta E=\left(\frac{n l}{2 \pi} \operatorname{Re} k\right)^{2}-\left[\left(m+\frac{2}{\pi} \delta\right)^{2}+p^{2}\right] .
$$

From this curve it follows that this difference tends to zero with $m$ increasing, thus confirming the existence of the term proportional to $p^{2}$. By fitting this difference with the simplest expression

$$
\delta E=\frac{c}{m}
$$

we find that $c \simeq-6.9$. By subtracting this correction term from the difference (20), one gets the curve indicated with filled circles in the inset of Fig. 7(b). The result is one order of magnitude smaller than the difference itself. 


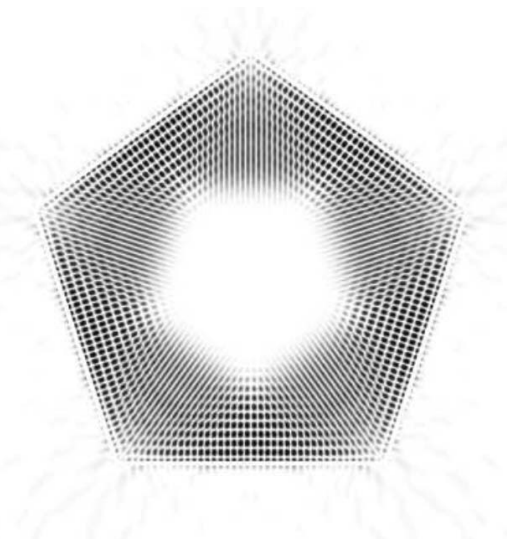

(a)

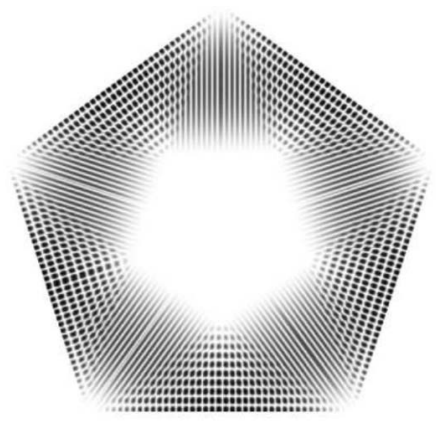

(d)

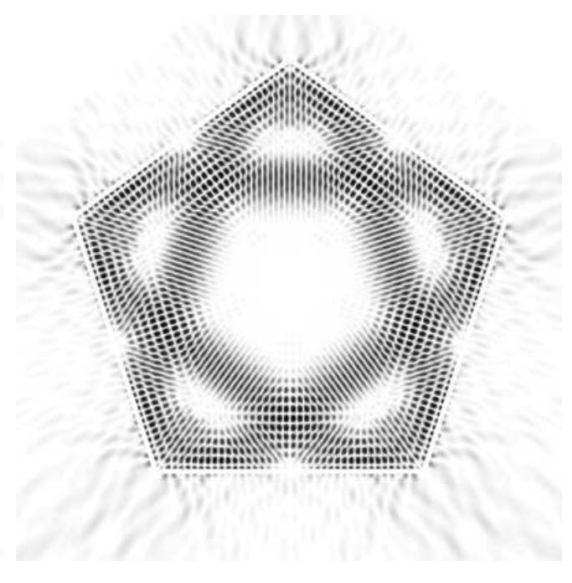

(b)

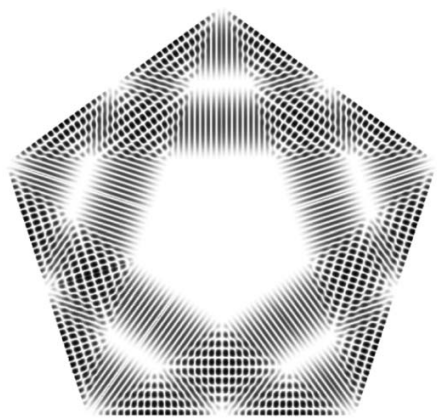

(e)

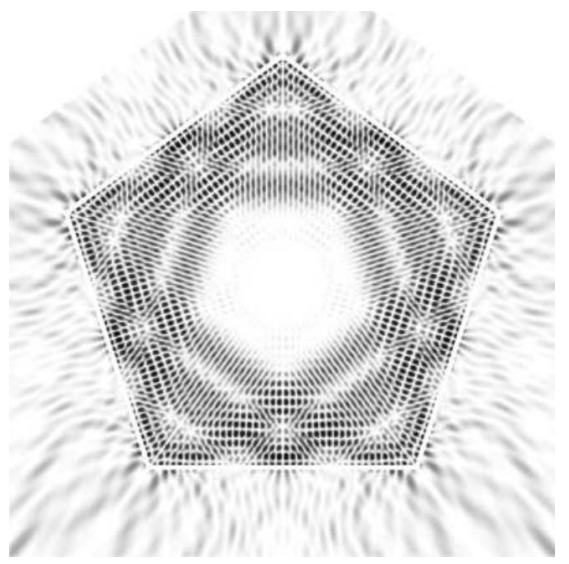

(c)

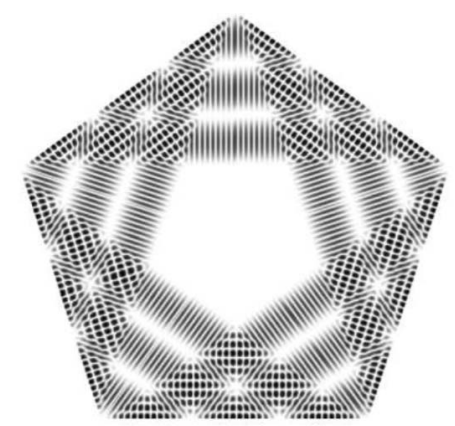

(f)

FIG. 13. (Top) Squared modulus of wave functions for pentagonal cavity with (- -) symmetry calculated with numerical simulations. (a) $a k=104.7-0.017 \mathrm{i}$, (b) $a k=102.2-0.05 \mathrm{i}$, (c) $a k=105.0-0.12 \mathrm{i}$. (Bottom) Squared modulus of wave functions calculated within the superscar model and corresponding to the paramters of the top pictures.

All these calculations confirm that the real parts of resonance wave numbers for square dielectric cavities are well reproduced in the semiclassical limit by the above superscar formula (19) and our experimental results can be considered as an implicit experimental confirmation of this statement.

\section{PENTAGONAL MICROCAVITY}

The trace formula and superscar model arguments can be generalized to all polygonal cavities. The pentagonal resonator provides a new interesting test. In fact, due to the odd number of sides, the inscribed pentagonal orbit [indicated by solid line in Fig. 10(a) ] is isolated. The shortest confined periodic orbit family is twice longer. It is represented with a dashed line in Fig. 10(a) and can be mapped onto the fivepointed star orbit drawn in Fig. 10(b) by continuous deformation. In this section we compare the predictions of the superscar model for this periodic orbit family with numerical simulations and experiments.

Due to the $C_{5 v}$ symmetry, pentagonal cavities sustain ten symmetry classes corresponding to the rotations by $2 \pi / 5$ and the inversion with respect to one of the symmetry axis. We have studied numerically one symmetry class in which wave functions obey the Dirichlet boundary conditions along two sides of a right triangle with angle $\pi / 5$ [see Fig. 10(a) in gray]. The results of these computations are presented in Fig. 11.

As for the square cavity, lowest loss states are organized in families. The wave functions of the three lowest loss families are plotted in the top of Fig. 13 and their superscar structure is obvious.

The computation of pure superscar states can be performed as in the previous section. The five-pointed star periodic orbit channel is shown in Fig. 12. In this case boundary conditions along horizontal boundaries of periodic orbit channel are not known. By analogy with superscar formation in polygonal billiards [24], we impose that wave functions tend to zero along these boundaries when $k \rightarrow \infty$.

Therefore a superscar wave function propagating inside this channel takes the form 


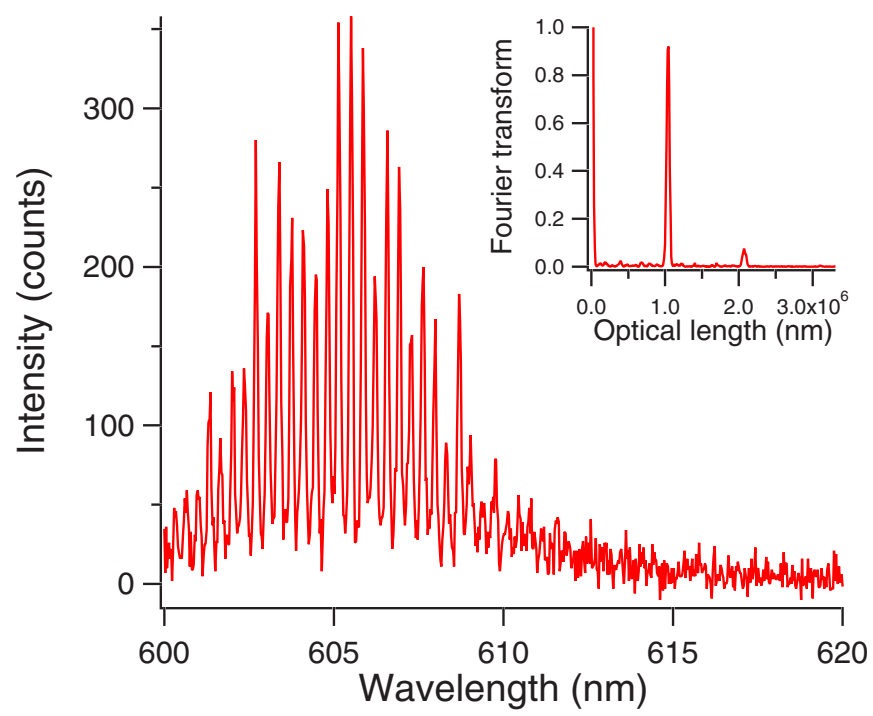

FIG. 14. (Color online) Experimental spectrum of a pentagonal microlaser of $80-\mu \mathrm{m}$ side length. Inset: Normalized Fourier transform of the spectrum expressed as intensity vs wave number.

$$
\Psi_{\text {scar }}(x, y)=\exp (i \kappa x) \sin \left(\frac{\pi}{w} p y\right) \Theta(y) \Theta(w-y),
$$

where $w$ is the width of the channel [for the five-pointed star orbit $w=a \sin (\pi / 5)$ where $a$ is the length of the pentagon side]. $\Theta(x)$ is the Heavyside function introduced here to stress that superscar functions are zero (or small) outside the periodic orbit channel.

The quantized values of the longitudinal momentum $\kappa$ are obtained by imposing that the function (22) is periodic along the channel when all phases due to the reflection with the cavity boundaries are taken into account

$$
\kappa L=2 \pi\left(M+\frac{10}{\pi} \delta\right)
$$

Here $M$ is an integer and $L$ is the total periodic orbit length. For the five-pointed star orbit (see Fig. 10)

$$
L=10 a \cos \left(\frac{\pi}{5}\right)
$$

and $\delta$ is the phase of the reflection coefficient given by Eq. (3) with $\nu=1$ (for TM polarization) and $\theta=3 \pi / 10$. For these states the real part of the wave number is the following:

$$
n L \operatorname{Re} k=2 \pi\left(M+\frac{10}{\pi} \delta\right)+\mathcal{O}\left(\frac{1}{M}\right) .
$$

Wave functions inside the cavity are obtained by folding back the scar function (22) and choosing the correct representation of the chosen symmetry class. When Dirichlet boundary conditions are imposed along two sides of a right triangle passing through the center of the pentagon (see Fig. 12) $M=5(2 m)$ if $p$ is odd and $M=5(2 m-1)$ if $p$ is even. Then, the wave function inside the triangle is the sum of two terms,

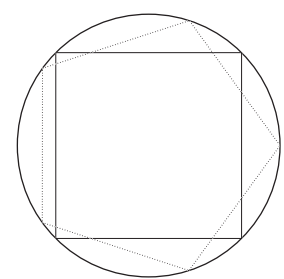

(a)

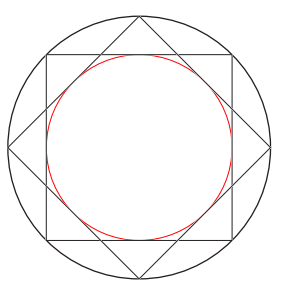

(b)
FIG. 15. (Color online) (a) Two examples of periodic orbits: the square and the pentagon. (b) Two representations of the square periodic orbit and the caustic of this family in red (inner circle).

$$
\begin{aligned}
\Psi_{m, p}(x, y)= & \sin \left(\kappa_{m} x\right) \sin \left(\frac{\pi}{w} p y\right) \Theta(y) \Theta(w-y) \\
& +\sin \left(k x^{\prime}-2 \delta\right) \sin \left(\frac{\pi}{w} p y^{\prime}\right) \Theta\left(y^{\prime}\right) \Theta\left(w-y^{\prime}\right),
\end{aligned}
$$

where the longitudinal momentum is

$$
\kappa_{m} \frac{L}{10}=2 \pi\left(m+\frac{1}{\pi} \delta-\xi\right)
$$

with $\xi=0$ for odd $p$ and $\xi=1 / 2$ for even $p$. $x^{\prime}$ and $y^{\prime}$ in Eq. (26) are coordinates of the point symmetric of $(x, y)$ with respect to the inversion on the edge of the pentagon. In the coordinate system when the pentagon edge passes through the origin (as in Fig. 12)

$$
x^{\prime}=x \cos 2 \phi+y \sin 2 \phi, \quad y^{\prime}=x \sin 2 \phi-y \cos 2 \phi,
$$

and $\phi=\pi / 5$ is the inclination angle of the pentagon side with respect to the abscissa axis. Wave functions obtained with this construction are presented in the bottom of Fig. 13. They correspond to the first, second, and third perpendicular excitations of the five-star periodic orbit family $(p=1,2$, and 3$)$.

To check the agreement between numerically computed real parts of the wave numbers and the superscar prediction (25) and (27), we plot in Fig. 11(b) the following difference:

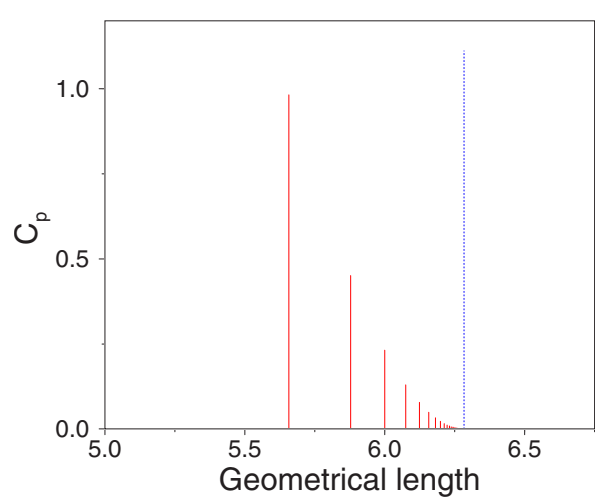

FIG. 16. (Color online) Vertical red sticks: $c_{p}$ coefficient for polygons confine by total internal reflection (square, pentagon, hexagon, etc.). The dotted blue line indicates the position of the perimeter. 

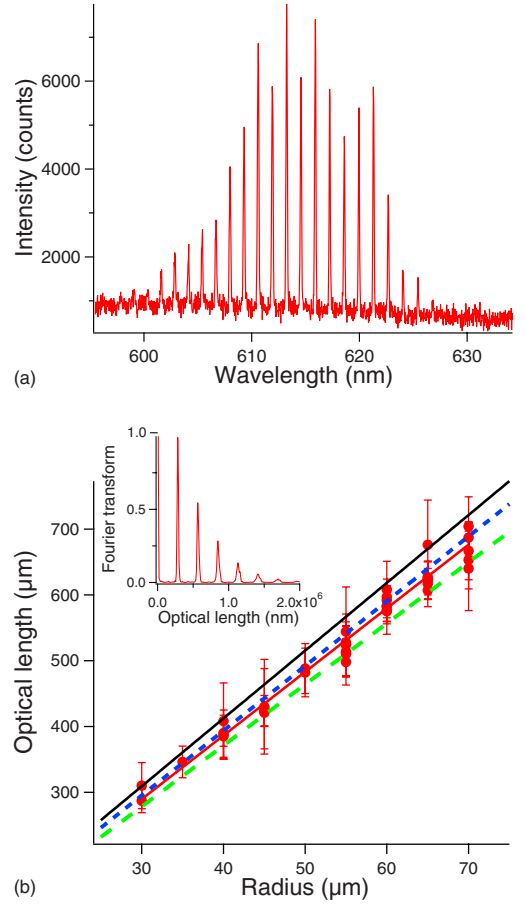

FIG. 17. (Color online) (a) Experimental spectrum of a microdisk of $30-\mu \mathrm{m}$ radius. (b) Optical length vs. radius. The experiments (red points) are linearly fitted by the sold red line. The other lines correspond to the theoretical predictions without any adjusted parameters: the dashed green line to the square, the dotted blue line to the hexagon, and the solid black line to the perimeter. Inset: Normalized Fourier transform of the spectrum in (a) expressed as intensity vs. wave number.

$$
\delta E=\left(\frac{a \cos }{2 \pi} \operatorname{Re} k a_{n u m}\right)^{2}-\left[\left(m+\frac{1}{\pi} \delta-\xi\right)^{2}+\left(\frac{l}{2 \tan \delta}\right)^{2} \zeta\right] .
$$

For pure scar states $\zeta=p^{2}$. As our numerical simulations have not reached the semiclassical limit (see scales in Figs. 7 and 11 ), we found it convenient to fit numerically the $\zeta$ constant. The best fit gives $\zeta \approx 0.44,2.33$, and 5.51 for the three most confined families (for pure scar functions this constant is 1 , 4,9 , respectively). The agreement is quite good with a relative accuracy of the order of $10^{-4}$ [see Fig. 11(b)]. Irrespective of the precise value of $\zeta$ the total optical length $n L$ is given by Eq. (25) and leads to an experimental prediction twice longer than the optical length of the inscribed pentagon, which is an isolated periodic orbit and thus cannot base superscar wave functions.

Comparison with experiments confirms the superscar nature of the most confined states for pentagonal resonators. In fact, the spectrum and its Fourier transform in Fig. 14 correspond to a pentagonal microlaser with side $a=80 \mu \mathrm{m}$, and show a periodic orbit with optical length $1040 \pm 30 \mu \mathrm{m}$ to be compared with the five-star optical length $n_{f u l l} 10 a \cos (\pi / 5)$ $=1061 \mu \mathrm{m}$. The agreement is better than $2 \%$.

This result is reproducible for cavities with the same size. Other sizes have been tested as well. For smaller cavities, the five-pointed star orbit is not identifiable due to lack of gain, whereas for bigger ones it is visible but mixed with nonconfined periodic orbits. This effect, not specific to pentagons, can be assigned to the contribution of different periodic orbit families which become important when the lasing gain exceeds the refractive losses. We will describe this phenomenon in a future publication [28].

The good agreement of numerical simulations and experiments with superscar predictions gives an additional credit to the validity of this approach even for nontrivial configurations.

\section{MICRODISKS}

Microdisk cavities are the simplest and most widely used microresonators. In the context of this work, they are of interest because of the coexistence of several periodic orbit families with close lengths. For low index cavities $(n \sim 1.5)$ each regular polygon trajectory with more than four sides is confined by total internal reflection.

In the two-dimensional approximation passive circular cavities are integrable and the spectrum of quasistationary states can be computed from an explicit quantization condition,

$$
n \frac{J_{m}^{\prime}(n k R)}{J_{m}(n k R)}=\nu \frac{H_{m}^{(1) \prime}(k R)}{H_{m}^{(1)}(k R)} .
$$

Here $R$ is the radius of the disk, $n$ is the refractive index of the cavity, and $\nu=1$ (respectively $\nu=n^{2}$ ) for the TM (respectively TE) polarization. For each angular quantum number $m$, an infinite sequence of solutions, $k_{m, q}$, is deduced from Eq. (29). They are labeled by the $q$ radial quantum number.

For large $|k|$ the $k_{m, l}$ wave numbers are obtained from a semiclassical expression (see, e.g., [29]) and the density of quasistationary states Eq. (15) can be proved to be rewritten as a sum over periodic orbit families. The derivation of this trace formula assumes only the semiclassical approximation $(|k| R \gg 1)$ and can be done in a way similar to that of the billiard case (see, e.g., [30]), leading to an expression closed to Eq. (16),

$$
d(k) \propto \sum_{p} \frac{A_{p}}{\sqrt{L_{p}}}\left|r_{p}\right|^{N_{p}} \cos \left[n L_{p} k-N_{p}\left(2 \delta_{p}+\frac{\pi}{2}\right)+\frac{\pi}{4}\right] .
$$

Here the $p$ index specifies a periodic orbit family. This formula depends on periodic orbit parameters: the number of bounces on the boundary, $N_{p}$, the incident angle on the boundary, $\chi_{p}$, the length, $L_{p}=2 N_{p} R \cos \left(\chi_{p}\right)$, and the area covered by periodic orbit family, $A_{p}=\pi R^{2} \cos ^{2}\left(\chi_{p}\right)$, which is the area included between the caustic and the boundary [see Fig. 15]. $2 \delta_{p}$ is the phase of the reflection coefficient at each bounce on the boundary [see Eq. (3)] and $\left|r_{p}\right|$ is its modulus.

For orbits confined by total internal reflection $\delta_{p}$ does not depend on $k R$ in the semiclassical limit, and $r_{p}$ is exponentially close to 1 [29,31]. From Eq. (30) it follows that each periodic orbit is singled out by a weighing coefficient $c_{p}=\frac{A_{p}}{\sqrt{L_{p}}}\left|r_{p}\right|^{N_{p}}$. Considering the experimental values $|k| R$ $\sim 1000,\left|r_{p}\right|$ can be approximated to unity with a good accu- 
racy for confined periodic orbits, and thus $c_{p}=\frac{A_{p}}{\sqrt{L_{p}}}$ depends only on geometrical quantities. Figure 16 shows the evolution of $c_{p}$ for polygons when the number of sides is increasing. As the critical angle is close to $45^{\circ}$, the diameter and triangle periodic orbits are not confined and the dominating contribution comes from the square periodic orbit. So we can reasonably conclude that the spectrum (15) of a passive twodimensional microdisk is dominated by the square periodic orbit.

The experimental method described in the previous sections has been applied to disk-shaped microcavities. A typical experimental spectrum is shown in Fig. 17(a). The first peak of its Fourier transform has a finite width coming from the experimental conditions (discretization, noise, etc.) and the contributions of several periodic orbits. This width is represented as error bars on the graph of Fig. 17(b). The continuous red line fitting the experimental data is surrounded by the dashed green line and the dotted blue line corresponding to the optical length of the square and hexagon, respectively, calculated with $n_{f u l l}=1.64$ as in the previous sections. The perimeter (continuous black line) overlaps with a large part of the error bars which evidences its contribution to the spectrum, but it is not close to experimental data.

These experimental results seem in good agreement with the above theoretical predictions. But actually these resonances, usually called whispering gallery modes, are living close to the boundary. Thus both roughness and threedimensional effects must be taken into account. At this stage it is difficult to evaluate and to measure correctly such contributions for each periodic orbit. For microdisks with a small thickness (about $0.4 \mu \mathrm{m}$ ) and designed with lower roughness, the results are more or less similar to those presented in Fig. 17(b).

\section{CONCLUSION}

We demonstrate experimentally that the length of the dominant periodic orbit can be recovered from the spectra of microlasers with simple shapes. Taking into account different dispersion corrections to the effective refractive index, a good agreement with theoretical predictions has been evidenced first for the Fabry-Perot resonator. Then we have tested polygonal cavities both with experiments and numerical simulations, and a good agreement for the real parts of wave numbers has been obtained even for the nontrivial configuration of the pentagonal cavity.

The observed dominance of confined short-period orbits is, in general, a consequence of the trace formula and the formation of long-lived states in polygonal cavities is related to strong diffraction on cavity corners. Finally, the study of microdisks highlights the case of several orbits and the influence of roughness and three-dimensional effect.

Our study opens the way to a systematic exploration of spectral properties by varying the shape of the boundary. In increasing the experimental precision even tiny details of trace formulas will be accessible. The improvement of the etching quality will suppress the leakage due to surface roughness and lead to a measure of the diffractive mode losses which should depend on symmetry classes. From the point of view of technology, it will allow a better prediction of the resonator design depending on the applications. From a more fundamental physics viewpoint, it may contribute to a better understanding of open dielectric billiards.

\section{ACKNOWLEDGMENTS}

The authors are grateful to S. Brasselet, R. Hierle, J. Lautru, C. T. Nguyen, and J.-J. Vachon for experimental and technological support and to C.-M. Kim, O. Bohigas, N. Sandeau, J. Szeftel, and E. Richalot for fruitful discussions.
[1] A. E. Siegman, Lasers (University Science Books, Mill Valley, CA, 1986).

[2] Optical Microcavities, edited by K. Vahala (World Scientific, Singapore, 2005).

[3] E. Krioukov, D. J. W. Klunder, A. Driessen, J. Greve, and C. Otto, Opt. Lett. 27, 512 (2002).

[4] A. M. Armani and K. J. Vahala, Opt. Lett. 31, 1896 (2006).

[5] C. Gmachl, F. Capasso, E. E. Narimanov, J. U. Nöckel, A. D. Stone, J. Faist, D. L. Sivco, and A. Y. Cho, Science 280, 1556 (1998).

[6] M. Lebental, J.-S. Lauret, J. Zyss, C. Schmit, and E. Bogomolny, Phys. Rev. A 75, 033806 (2007).

[7] R. C. Polson, G. Levina, and Z. V. Vardeny, Appl. Phys. Lett. 76, 3858 (2000).

[8] D. K. Armani, T. J. Kippenberg, S. M. Spillane, and K. J. Vahala, Nature (London) 421, 925 (2003).

[9] M. Lebental, J.-S. Lauret, R. Hierle, and J. Zyss, Appl. Phys. Lett. 88, 031108 (2006).

[10] C. Vassallo, Optical Waveguide Concepts (Elsevier, Amsterdam, 1991).
[11] P. K. Tien, Appl. Opt. 10, 2395 (1971).

[12] For some samples, the underlying layer is silica with refractive index $n_{2}=1.45$, so $n_{\text {eff }}$ is slightly different.

[13] This definition is consistent all over the paper. In the literature, these names are sometimes permutated.

[14] D. S. Jones, Proc. R. Soc. London, Ser. A 217, 153 (1953).

[15] T. Harayama, P. Davis, and K. S. Ikeda, Phys. Rev. Lett. 90, 063901 (2003).

[16] H. E. Türeci, A. D. Stone, and B. Collier, Phys. Rev. A 74, 043822 (2006)

[17] H. E. Türeci, A. D. Stone, and Li Ge, e-print arXiv:cond-mat/ 0610229.

[18] S. Shinohara, T. Harayama, H. E. Türeci, and A. D. Stone, Phys. Rev. A 74, 033820 (2006).

[19] C. Y. Fong and A. Poon, Opt. Express 12, 4864 (2004).

[20] Chaos and Quantum Physics, Les Houches Summer School Lectures LII, edited by M. J. Giannoni, A. Voros, and J. ZinnJustin (North-Holland, Amsterdam, 1991).

[21] R. Blümel, T. M. Antonsen, Jr., B. Georgeot, E. Ott, and R. E. Prange, Phys. Rev. E 53, 3284 (1996). 
[22] J. Wiersig, Phys. Rev. A 67, 023807 (2003).

[23] I. Braun, G. Ihlein, F. Laeri, J. U. Nöckel, G. Schulz-Ekloff, F. Schüth, U. Vietze, O. Weiß, and D. Wöhrle, Appl. Phys. B: Lasers Opt. 70, 335 (2000).

[24] E. Bogomolny and C. Schmit, Phys. Rev. Lett. 92, 244102 (2004).

[25] E. Bogomolny, B. Dietz, T. Friedrich, M. Miski-Oglu, A. Richter, F. Schäfer, and C. Schmit, Phys. Rev. Lett. 97, 254102 (2006).

[26] For a given symmetry class, the length entering the quantization condition may be a part of the total periodic orbit length.
[27] The estimation of the imaginary parts of these states as well as the field distribution outside the cavity is beyond the scope of this paper and will be discussed elsewhere.

[28] M. Lebental, N. Djellali, J.-S. Lauret, J. Zyss, R. Dubertrand, C. Schmit, and E. Bogomolny (to be published).

[29] J. Nöckel, Ph.D. dissertation, Yale University, 1997.

[30] M. Brack and R. K. Bhaduri, Semiclassical Physics (AddisonWesley, Reading MA, 1997).

[31] H. Schomerus and M. Hentschel, Phys. Rev. Lett. 96, 243903 (2006). 